\title{
On the Darboux problem involving the distributional Henstock-Kurzweil integral
}

\author{
Guoju Ye', Rong Cheng ${ }^{1 *}$, Wei Liu' and Hui Mei²
}

"Correspondence:

chengr94@163.com

${ }^{1}$ College of Science, Hohai

University, Nanjing, China

Full list of author information is

available at the end of the article

\begin{abstract}
In this paper, we apply the method associated with the technique of measure of noncompactness and the Darbo fixed point theorem to study the existence of solutions of the Darboux problem involving the distributional Henstock-Kurzweil integral. Meanwhile, an example is provided to illustrate our results.
\end{abstract}

MSC: Primary 47H08; secondary 26A39

Keywords: Darboux problem; Distributional Henstock-Kurzweil integral; Measure of noncompactness; Darbo fixed point theorem

\section{Introduction}

The Darboux problems have been studied by many authors (see [1-3]). In [2], the authors used a fixed point theorem and some properties of measure of weak noncompactness to prove the existence of pseudo-solutions for the Darboux problem in a Banach space $E$ :

$$
\left\{\begin{array}{l}
\frac{\partial^{2} u}{\partial x \partial y}(x, y)=g(x, y, u(x, y)), \quad(x, y) \in \Delta, \\
u(x, 0)=0 \\
u(0, y)=0
\end{array}\right.
$$

where $\Delta=\left\{(x, y): 0 \leq x \leq a_{1}, 0 \leq y \leq a_{2}\right\}, g$ is Pettis-integrable but not necessarily Bochner integrable, and $\frac{\partial^{2} u}{\partial x \partial y}$ denotes the second-order mixed pseudo-derivative.

In this paper, instead of the pseudo-derivative, we use the distributional derivative to establish the existence of solutions for the following Darboux problem involving the distributional Henstock-Kurzweil integral ( $D_{\mathrm{HK}}$-integral):

$$
\left\{\begin{array}{l}
\partial_{x y} u(x, y)=g(x, y, u(x, y))+f(x, y), \quad(x, y) \in \Delta \\
u(x, 0)-\psi_{1}(x, u)=h_{1}(y) \\
u(0, y)-\psi_{2}(y, u)=h_{2}(x)
\end{array}\right.
$$

where $\partial_{x y}$ denotes the second-order mixed distributional derivative, $g$ is HenstockKurzweil integral ( $H K$-integral), $\psi_{i}, h_{i}$ are continuous, $f$ is $D_{\mathrm{HK}}$-integrable.

If there exists a continuous function $F \in C_{0}(\bar{Q})$ on $\bar{Q}$ such that $f$ is the distributional derivative of $F$ (the definition of $C_{0}(\bar{Q})$ will be introduced in Sect. 2), then the distribution

(c) The Author(s) 2018. This article is distributed under the terms of the Creative Commons Attribution 4.0 International License (http://creativecommons.org/licenses/by/4.0/), which permits unrestricted use, distribution, and reproduction in any medium, provided you give appropriate credit to the original author(s) and the source, provide a link to the Creative Commons license, and indicate if changes were made. 
$f$ is $D_{\mathrm{HK}}$-integrable on $Q$. In other words, $F$ is the primitive function of $f$. So we can see from the definition of $D_{\mathrm{HK}}$-integral that the $D_{\mathrm{HK}}$-integral is a kind of integration that is more extensive than the Riemann integral, Lebesgue integral, Henstock-Kurzweil integral and Denjoy integral (see [4-9]).

In order to prove the existence of solutions of the Darboux problem involving the distributional Henstock-Kurzweil integral, the method associated with the technique of measure of noncompactness and the Darbo fixed point theorem will be used.

This paper is organized as follows. In Sect. 2, we recall some fundamental concepts and basic results of the $D_{\mathrm{HK}}$-integral and measure of noncompactness. In Sect. 3, we apply the Darbo fixed point theorem [10, Theorem 2] related to measure of noncompactness to verify the existence of solutions of Eq. (2). In Sect. 4, we give an example to illustrate Theorem 3.3 in this paper.

\section{Preliminaries}

In this section, we provide preliminary material with respect to the $D_{\mathrm{HK}}$-integral and the measure of noncompactness.

\section{1 $D_{\mathrm{HK}}$ integral}

Let $Q$ be an open rectangle $(a, b) \times(c, d)$ in the plane $\mathbb{R}^{2}$, and $\mathcal{D}(Q)$ be a subset of $C^{\infty}(Q)$ such that each $\phi \in \mathcal{D}(Q)$ has a compact support in $Q$. The continuous linear functional on $\mathcal{D}(Q)$ is called a distribution on $Q$.

We denote by $\partial=\partial_{x y}=\partial_{y x}$ the mixed distributional derivative, by $\partial_{1}$ and $\partial_{2}$ the distributional derivatives with respect to $x$ and $y$, respectively, and by ' $\int$ ' the $D_{\mathrm{HK}}$-integral.

Let

$$
C_{0}(\bar{Q})=\{F \in C(\bar{Q}): F(a, y)=F(x, c)=0 \text { for }(x, y) \in \bar{Q}\}
$$

where $\bar{Q}$ is the closure of $Q$.

Obviously, $C_{0}(\bar{Q})$ is a closed subspace of $C(\bar{Q})$ endowed with the norm $\|F\|_{\infty}=$ $\max \{|F(x, y)|:(x, y) \in \bar{Q}, F(x, y) \in C(\bar{Q})\}$.

We define the distributional Henstock-Kurzweil integral:

$$
D_{\mathrm{HK}}(Q)=\left\{f \in \mathcal{D}^{\prime}(Q) \mid f=\partial F, F \in C_{0}(\bar{Q})\right\} .
$$

Definition 2.1 ([11, Lemma 2]) If $f \in \mathcal{D}^{\prime}(Q)$, then

$$
\begin{aligned}
& F_{1}(x, y)+F_{1}(a, c)-F_{1}(a, y)-F_{1}(x, c) \\
& \quad=F_{2}(x, y)+F_{2}(a, c)-F_{2}(a, y)-F_{2}(x, c)
\end{aligned}
$$

for all $F_{1}, F_{2} \in C_{0}(\bar{Q}),(x, y) \in \bar{Q}$. Moreover, there exists a unique $F_{f} \in D_{\mathrm{HK}}(Q)$ such that

$$
F_{f}(a, y)=F_{f}(x, c)=0,
$$

$\forall x \in[a, b], y \in[c, d]$.

This leads to the next definition. 
Definition 2.2 A distribution $f$ is $D_{\mathrm{HK}}$-integrable on $Q$ if $f \in D_{\mathrm{HK}}(Q)$.

The $D_{\mathrm{HK}}$-integral of $f$ on $Q$ is given by $\int_{Q} f=F_{f}(b, d)$, where $F_{f}$ is given by Definition 2.1 . We consider the structure of space $D_{\mathrm{HK}}(Q)$. For $f \in D_{\mathrm{HK}}(Q)$, endowed with the norm

$$
\|f\|=\sup \left\{\left|\int_{(a, x) \times(c, y)} f\right|:(x, y) \in \bar{Q}\right\} .
$$

Lemma $2.3([11$, Theorem 1$])$ The normed space $\left(D_{\mathrm{HK}}(Q),\|\cdot\|\right)$ is complete, separable and isomorphic to $\left(C_{0}(\bar{Q}),\|\cdot\|_{\infty}\right)$.

Before stating a Fubini-type theorem for $D_{\mathrm{HK}}$-integral which will be used later, we introduce some definitions.

Definition 2.4 Let $f \in D_{\mathrm{HK}}(Q), x \in[a, b], y \in[c, d]$. We define

$$
\begin{aligned}
& \int_{a}^{x} f(\xi, \cdot) d \xi=\partial_{2} F_{f}(x, \cdot) \quad \text { in } \mathcal{D}^{\prime}((c, d)), \\
& \int_{c}^{y} f(\cdot, \eta) d \eta=\partial_{1} F_{f}(\cdot, y) \quad \text { in } \mathcal{D}^{\prime}((a, b)) .
\end{aligned}
$$

It is clear that

$$
\int_{a}^{x} f(s, \cdot) d s \in D_{\mathrm{HK}}((c, d)), \quad \int_{c}^{y} f(\cdot, t) d t \in D_{\mathrm{HK}}((a, b)),
$$

where $D_{\mathrm{HK}}((a, b))$ and $D_{\mathrm{HK}}((c, d))$ are, respectively, the spaces of $D_{\mathrm{HK}}$-integrable distributions on $(a, b)$ and $(c, d)$, i.e.

$$
D_{\mathrm{HK}}((a, b))=\left\{f \in \mathcal{D}^{\prime}((a, b)) \mid f=\partial F, F \in C_{0}(\bar{Q})\right\},
$$

where $C_{0}(\bar{Q})=\{F \in C([a, b]): F(a)=0\}$, and $f$ is the distributional derivative of $F$.

Lemma 2.5 ([11, Theorem 4]) For all $f \in D_{\mathrm{HK}}(Q)$, we have

$$
\int_{Q} f=\int_{a}^{b}\left(\int_{c}^{d} f(\cdot, \eta) d \eta\right)=\int_{c}^{d}\left(\int_{a}^{b} f(\xi, \cdot) d \xi\right) .
$$

Lemma 2.6 ([4, Theorem 4.3]) If the following conditions are satisfied:

(i) $f_{n}(x) \rightarrow f(x)$ almost everywhere in $[a, b]$ as $n \rightarrow \infty$ where each $f_{n}$ is Henstock integrable on $[a, b]$.

(ii) $g(x) \leq f_{n}(x) \leq h(x)$ for almost all $x \in[a, b]$ and all $n$. If $g$ and $h$ are also Henstock integrable on $[a, b]$,

then $f$ is Henstock integrable on $[a, b]$ and

$$
\int_{a}^{b} f_{n} \rightarrow \int_{a}^{b} f \text { as } n \rightarrow \infty
$$




\subsection{Measure of noncompactness}

In this subsection, we recall the definition and some basic properties concerning measure of noncompactness [10]. Let $(E,\|\cdot\|)$ be a real Banach space with zero element $0, B(x, r)$ be the closed ball in $E$ centered at $x$ and of radius $r$, and $B_{r}$ be the ball $B(0, r)$. Denote by $\bar{X}, \overline{\operatorname{conv}} X$ the closure and the closed convex hull of a nonempty subset $X$ of $E$, respectively. Finally, denote by $\mathfrak{m}_{E}$ and $\mathfrak{n}_{E}$ the family of all nonempty and bounded subsets of $E$ and the subfamilies of all relatively compact subsets, respectively.

Definition 2.7 ([12]) Let $(E, d)$ be a metric space and $X$ a bounded subset of $E$. The Hausdorff measure of noncompactness ( $\mu$-measure or ball measure of noncompactness) of the set $X$, denoted by $\mu(X)$ is defined to be the infimum of the set of all reals $\varepsilon>0$ such that $X$ can be covered by a finite number of balls of sets with diameters $<\varepsilon$, that is,

$$
\mu(X)=\inf \{\varepsilon>0: X \text { has a finite } \varepsilon \text {-net in } E\} .
$$

The function $\mu$ is called the Hausdorff measure of noncompactness.

Definition 2.8 ([10]) A mapping $\mu: \mathfrak{m}_{E} \rightarrow \mathbb{R}_{+}$is said to be a measure of noncompactness in $E$ if it satisfies the following conditions:

(1) The family $\operatorname{ker} \mu=\left\{X \in \mathfrak{m}_{E}: \mu(X)=0\right\}$ is nonempty and ker $\mu \subseteq \mathfrak{n}_{E}$.

(2) if $X \subseteq Y \Rightarrow \mu(X) \leq \mu(Y)$.

(3) $\mu(\overline{\operatorname{conv}} X)=\mu(X)=\mu(\bar{X})$.

(4) $\mu(\lambda X+(1-\lambda) Y) \leq \lambda \mu(X)+(1-\lambda) \mu(Y)$ for $\lambda \in[0,1)$.

(5) If $\left(X_{n}\right)$ is a sequence of closed sets from $\mathfrak{m}_{E}$ such that $X_{n+1} \subseteq X_{n}(n \geq 1)$ and if $\lim _{n \rightarrow \infty} \mu\left(X_{n}\right)=0$, then the intersection set $X_{\infty}=\bigcap_{n=1}^{\infty} X_{n}$ is nonempty.

In what follows, we will work in the space $C(\Delta)$ consisting of all real valued continuous functions on $\Delta$. The space $C(\Delta)$ is equipped with the supremum norm

$$
\|u\|=\sup \{|u(x, y)|:(x, y) \in \Delta\} .
$$

For each $u \in C(\Delta)$, we define

$$
\omega(u, \varepsilon)=\sup \left\{\left|u\left(x_{2}, y_{2}\right)-u\left(x_{1}, y_{1}\right)\right|:\left|x_{2}-x_{1}\right| \leq \varepsilon,\left|y_{2}-y_{1}\right| \leq \varepsilon\right\} .
$$

Obviously, $\omega(u, \varepsilon) \rightarrow 0$, as $\varepsilon \rightarrow 0$, since $u$ is uniformly continuous on $\Delta$. Moreover, if this limit relation holds uniformly for $u$ running over some bounded set $X \subset C(\Delta)$, then $X$ is equicontinuous, and vice versa. Therefore, we have the following.

Lemma 2.9 ([13, Theorem 2.2]) On the space $C(\Delta)$, the measure of noncompactness (3) is equivalent to

$$
\mu(X)=\lim _{\varepsilon \rightarrow 0} \sup _{u \in X} \omega(u, \varepsilon)
$$


Lemma 2.10 ([10, Theorem 2, Darbo]) Let $\Omega$ be a nonempty, bounded, closed and convex subset of a Banach space E and let $T: \Omega \rightarrow \Omega$ be a continuous mapping. Assume that there exists a constant $k \in[0,1)$ such that

$$
\mu(T(X)) \leq k \mu(X)
$$

for any $X \subset \Omega$. Then $T$ has a fixed point.

\section{Main results}

In this section, we shall prove the existence of solutions of Eq. (2).

Firstly, we give the following assumptions:

$\left(D_{1}\right)$ The functions $\psi_{i}:\left[0, a_{i}\right] \times \mathbb{R} \rightarrow \mathbb{R}$ are continuous, and there exist nonnegative constants $c_{i}>0(i=1,2)$ such that, for each $u, v \in C(\Delta)$,

$$
\begin{aligned}
& \left|\psi_{1}(x, u)-\psi_{1}(x, v)\right| \leq c_{1}(|u-v|), \\
& \left|\psi_{2}(y, u)-\psi_{2}(y, v)\right| \leq c_{2}(|u-v|), \quad c_{1}+c_{2}<1 .
\end{aligned}
$$

Let $M_{1}=\sup \left\{\left|\psi_{1}(x, 0)\right|: x \in\left[0, a_{1}\right]\right\}, M_{2}=\sup \left\{\left|\psi_{2}(y, 0)\right|: y \in\left[0, a_{2}\right]\right\}$.

$\left(D_{2}\right)$ The functions $h_{1}:\left[0, a_{2}\right] \rightarrow \mathbb{R}, h_{2}:\left[0, a_{1}\right] \rightarrow \mathbb{R}$ are continuous, and $h_{1}(0)=h_{2}(0)$, let $M_{3}=\sup \left\{\left|h_{1}(y)\right|: y \in\left[0, a_{2}\right]\right\}, M_{4}=\sup \left\{\left|h_{2}(x)\right|: x \in\left[0, a_{1}\right]\right\}$.

$\left(D_{3}\right)$ The function $f$ is $D_{\mathrm{HK}}$-integrable on $\Delta$, and $M_{5}=\sup _{(x, y) \in \Delta}\left\{\left|\int_{0}^{x} \int_{0}^{y} f(s, t) d t d s\right|\right\}$.

$\left(D_{4}\right)$ The function $g: \Delta \times \mathbb{R} \rightarrow \mathbb{R}$ is $H K$-integrable, for each $(x, y) \in \Delta, z \mapsto g(x, y, z)$ is continuous, and there exists $H K$-integral function $g_{-}, g_{+}: \Delta \rightarrow \mathbb{R}$ such that

$$
g_{1}(\cdot, \cdot) \leq g(\cdot, \cdot, z) \leq g_{+}(\cdot, \cdot)
$$

and $M_{6}=\sup _{(x, y) \in \Delta}\left\{\left|\int_{0}^{x} \int_{0}^{y} g_{-}(s, t) d t d s\right|+\left|\int_{0}^{x} \int_{0}^{y} g_{+}(s, t) d t d s\right|\right\}$.

$\left(D_{5}\right)$ There exists $r>0$ such that

$$
M_{1}+M_{2}+2 M_{3}+M_{4}+M_{5}+M_{6}+\left(c_{1}+c_{2}\right) r \leq r .
$$

Theorem 3.1 Under the assumptions $\left(D_{1}\right)-\left(D_{5}\right)$, Eq. (2) is equivalent to the integral equation

$$
\begin{aligned}
u(x, y)= & \psi_{1}(x, u)+h_{1}(y)+\psi_{2}(y, u)+h_{2}(x)-h_{1}(0)+\int_{0}^{x} \int_{0}^{y} f(s, t) d t d s \\
& +\int_{0}^{x} \int_{0}^{y} g(s, t, u(s, t)) d t d s, \quad(x, y) \in \Delta .
\end{aligned}
$$

Proof For all $(x, y) \in \Delta$, by the properties of the distributional derivative, we have

$$
\int_{0}^{y} \partial_{s t} u(s, t) d t=\int_{0}^{y} g(s, t, u(s, t)) d t+\int_{0}^{y} f(s, t) d t .
$$

Since

$$
\partial_{s} u(s, y)-\partial_{s} u(s, 0)=\int_{0}^{y} g(s, t, u(s, t)) d t+\int_{0}^{y} f(s, t) d t,
$$


then

$$
\int_{0}^{x} \partial_{s} u(s, y) d s=\int_{0}^{x} \partial_{s} u(s, 0) d s+\int_{0}^{x} \int_{0}^{y} g(s, t, u(s, t)) d t d s+\int_{0}^{x} \int_{0}^{y} f(s, t) d t d s .
$$

We obtain

$$
\begin{aligned}
u(x, y)= & u(x, 0)+u(0, y)-u(0,0)+\int_{0}^{x} \int_{0}^{y} g(s, t, u(s, t)) d t d s+\int_{0}^{x} \int_{0}^{y} f(s, t) d t d s \\
= & \psi_{1}(x, u)+h_{1}(y)+\psi_{2}(y, u)+h_{2}(x)-h_{1}(0)+\int_{0}^{x} \int_{0}^{y} f(s, t) d t d s \\
& +\int_{0}^{x} \int_{0}^{y} g(s, t, u(s, t)) d t d s .
\end{aligned}
$$

On the other hand, it is not difficult to see that Eq. (2) holds by taking the derivative of both sides of (9). This completes the proof.

To simplify, we define an operator $F$ on $C(\Delta)$ by

$$
\begin{aligned}
F u(x, y)= & \psi_{1}(x, u)+h_{1}(y)+\psi_{2}(y, u)+h_{2}(x)-h_{1}(0)+\int_{0}^{x} \int_{0}^{y} f(s, t) d t d s \\
& +\int_{0}^{x} \int_{0}^{y} g(s, t, u(s, t)) d t d s, \quad(x, y) \in \Delta .
\end{aligned}
$$

Then we have the following statement.

Theorem 3.2 Under the assumptions $\left(D_{1}\right)-\left(D_{5}\right)$, the operator F given in (10) has at least one fixed point in the space $C(\Delta)$.

Proof (i) For any $u \in C(\Delta)$, with $\|u\| \leq r$,

$$
\begin{aligned}
|F u(x, y)| \leq & \left|\psi_{1}(x, u)\right|+\left|h_{1}(y)\right|+\left|\psi_{2}(y, u)\right|+\left|h_{2}(x)\right|+\left|h_{1}(0)\right|+\left|\int_{0}^{x} \int_{0}^{y} f(s, t) d t d s\right| \\
& +\left|\int_{0}^{x} \int_{0}^{y} g(s, t, u(s, t)) d t d s\right| \\
\leq & \left|\psi_{1}(x, u)-\psi_{1}(x, 0)\right|+\left|\psi_{1}(x, 0)\right|+M_{3}+\left|\psi_{2}(y, u)-\psi_{2}(y, 0)\right|+\left|\psi_{2}(y, 0)\right| \\
& \quad+M_{4}+M_{3}+M_{5}+\left|\int_{0}^{x} \int_{0}^{y} g_{-}(s, t) d t d s\right|+\left|\int_{0}^{x} \int_{0}^{y} g_{+}(s, t) d t d s\right| \\
\leq & M_{1}+M_{2}+2 M_{3}+M_{4}+M_{5}+M_{6}+\left(c_{1}+c_{2}\right)\|u\| \\
\leq & r .
\end{aligned}
$$

This implies that $F$ maps the space $B_{r}$ into $B_{r}$, where $B_{r}=\{u \in C(\Delta):\|u\| \leq r\}, r$ is a constant appearing in assumption $\left(D_{5}\right)$.

(ii) We prove that the operator $F$ is continuous on $B_{r}$. For arbitrary $u \in B_{r}$ and $\varepsilon>0$, now let $u_{n} \in B_{r}$ with $\left\|u_{n}-u\right\|<\varepsilon$, then we have

$$
\left|F u_{n}(x, y)-F u(x, y)\right|
$$




$$
\begin{aligned}
\leq & \left|\psi_{1}\left(x, u_{n}\right)-\psi_{1}(x, u)\right|+\left|\psi_{2}\left(y, u_{n}\right)-\psi_{2}(y, u)\right| \\
& +\left|\int_{0}^{x} \int_{0}^{y} g\left(s, t, u_{n}(s, t)\right) d t d s-\int_{0}^{x} \int_{0}^{y} g(s, t, u(s, t)) d t d s\right| .
\end{aligned}
$$

Now by the uniform continuity of the function $\psi_{i}(i=1,2)$ on the set $\left[0, a_{i}\right] \times[-r, r]$, we infer that

$$
\lim _{n \rightarrow \infty} \psi_{1}\left(x, u_{n}\right)=\psi_{1}(x, u), \lim _{n \rightarrow \infty} \psi_{2}\left(y, u_{n}\right)=\psi_{2}(y, u)
$$

According to $\left(D_{4}\right)$ and Lemma 2.6, we have

$$
\lim _{n \rightarrow \infty} \int_{0}^{x} \int_{0}^{y} g\left(s, t, u_{n}(s, t)\right) d t d s=\int_{0}^{x} \int_{0}^{y} g(s, t, u(s, t)) d t d s
$$

From estimate (11), for each $(x, y) \in \Delta$, we get

$$
\left|F u_{n}(x, y)-F u(x, y)\right| \leq \varepsilon .
$$

Hence, we conclude that the operator $F$ is continuous on $B_{r}$.

(iii) Let us take an arbitrary nonempty subset $V$ of the ball $B_{r}$. Fix $\varepsilon>0$, choose arbitrarily $\left(x_{1}, y_{1}\right),\left(x_{2}, y_{2}\right) \in \Delta$ such that $\left|x_{2}-x_{1}\right| \leq \varepsilon,\left|y_{2}-y_{1}\right| \leq \varepsilon$. Then for arbitrary $u \in V$, we get

$$
\begin{aligned}
\left|F u\left(x_{2}, y_{2}\right)-F u\left(x_{1}, y_{1}\right)\right| & \\
\leq & \left|\psi_{1}\left(x_{2}, u\left(x_{2}, y_{2}\right)\right)-\psi_{1}\left(x_{1}, u\left(x_{1}, y_{1}\right)\right)\right|+\left|h_{1}\left(y_{2}\right)-h_{1}\left(y_{1}\right)\right| \\
& +\left|\psi_{2}\left(y_{2}, u\left(x_{2}, y_{2}\right)\right)-\psi_{2}\left(y_{1}, u\left(x_{1}, y_{1}\right)\right)\right|+\left|h_{2}\left(x_{2}\right)-h_{2}\left(x_{1}\right)\right| \\
& +\left|\int_{0}^{x_{2}} \int_{0}^{y_{2}} f(s, t) d t d s-\int_{0}^{x_{1}} \int_{0}^{y_{1}} f(s, t) d t d s\right| \\
& +\left|\int_{0}^{x_{2}} \int_{0}^{y_{2}} g(s, t, u(s, t)) d t d s-\int_{0}^{x_{1}} \int_{0}^{y_{1}} g(s, t, u(s, t)) d t d s\right| \\
\leq & \left|\psi_{1}\left(x_{2}, u\left(x_{2}, y_{2}\right)\right)-\psi_{1}\left(x_{1}, u\left(x_{2}, y_{2}\right)\right)\right|+\left|\psi_{1}\left(x_{1}, u\left(x_{2}, y_{2}\right)\right)-\psi_{1}\left(x_{1}, u\left(x_{1}, y_{1}\right)\right)\right| \\
& +\omega\left(h_{1}, \varepsilon\right)+\left|\psi_{2}\left(y_{2}, u\left(x_{2}, y_{2}\right)\right)-\psi_{2}\left(y_{1}, u\left(x_{2}, y_{2}\right)\right)\right| \\
& +\left|\psi_{2}\left(y_{1}, u\left(x_{2}, y_{2}\right)\right)-\psi_{2}\left(y_{1}, u\left(x_{1}, y_{1}\right)\right)\right|+\omega\left(h_{2}, \varepsilon\right) \\
& +\left|\int_{0}^{x_{1}} \int_{y_{1}}^{y_{2}} f(s, t) d t d s+\int_{x_{1}}^{x_{2}} \int_{0}^{y_{2}} f(s, t) d t d s\right| \\
& +\left|\int_{0}^{x_{1}} \int_{y_{1}}^{y_{2}} g(s, t, u(s, t)) d t d s+\int_{x_{1}}^{x_{2}} \int_{0}^{y_{2}} g(s, t, u(s, t)) d t d s\right| \\
\leq & \omega\left(\psi_{1}, \varepsilon\right)+c_{1}\left(\left|u\left(x_{2}, y_{2}\right)-u\left(x_{1}, y_{1}\right)\right|\right)+\omega\left(h_{1}, \varepsilon\right)+\omega\left(\psi_{2}, \varepsilon\right) \\
+ & c_{2}\left(\left|u\left(x_{2}, y_{2}\right)-u\left(x_{1}, y_{1}\right)\right|\right)+\omega\left(h_{2}, \varepsilon\right) \\
+ & \left|\int_{y_{1}}^{x_{1}} \int_{y_{1}}^{y_{2}} f(s, t) d t d s+\int_{x_{1}}^{x_{2}} g(s, t, u(s, t)) d t d s+\int_{0}^{x_{2}} g(s, t, u(s, t)) d t d s\right| \\
& +\mid
\end{aligned}
$$


where

$$
\begin{aligned}
& \omega\left(\psi_{1}, \varepsilon\right)=\sup \left\{\left|\psi_{1}\left(x_{2}, u\right)-\psi_{1}\left(x_{1}, u\right)\right|: u \in[-r, r],\left|x_{2}-x_{1}\right| \leq \varepsilon\right\}, \\
& \omega\left(h_{1}, \varepsilon\right)=\sup \left\{\left|h_{1}\left(y_{2}\right)-h_{1}\left(y_{1}\right)\right|:\left|y_{2}-y_{1}\right| \leq \varepsilon\right\}, \\
& \omega\left(\psi_{2}, \varepsilon\right)=\sup \left\{\left|\psi_{2}\left(y_{2}, u\right)-\psi_{2}\left(y_{1}, u\right)\right|: u \in[-r, r],\left|y_{2}-y_{1}\right| \leq \varepsilon\right\}, \\
& \omega\left(h_{2}, \varepsilon\right)=\sup \left\{\left|h_{2}\left(x_{2}\right)-h_{2}\left(x_{1}\right)\right|:\left|x_{2}-x_{1}\right| \leq \varepsilon\right\} .
\end{aligned}
$$

By $\left(D_{1}\right)-\left(D_{2}\right), \psi_{i}(i=1,2), h_{1}, h_{2}$ are uniformly continuous on $\left[0, a_{i}\right],\left[0, a_{2}\right],\left[0, a_{1}\right]$ respectively, so

$$
\omega\left(\psi_{i}, \varepsilon\right) \rightarrow 0, \quad \omega\left(h_{i}, \varepsilon\right) \rightarrow 0 \quad \text { as } \varepsilon \rightarrow 0
$$

Further, by the condition $\left(D_{4}\right)$, we have the following inequalities:

$$
\begin{gathered}
\left|\int_{0}^{x_{1}} \int_{y_{1}}^{y_{2}} g(s, t, u(s, t)) d t d s+\int_{x_{1}}^{x_{2}} \int_{0}^{y_{2}} g(s, t, u(s, t)) d t d s\right| \\
\leq\left|\int_{0}^{x_{1}} \int_{y_{1}}^{y_{2}} g_{-}(s, t) d t d s\right|+\left|\int_{0}^{x_{1}} \int_{y_{1}}^{y_{2}} g_{+}(s, t) d t d s\right| \\
\quad+\left|\int_{x_{1}}^{x_{2}} \int_{0}^{y_{2}} g_{-}(s, t) d t d s\right|+\left|\int_{x_{1}}^{x_{2}} \int_{0}^{y_{2}} g_{+}(s, t) d t d s\right| .
\end{gathered}
$$

Since $g_{-}, g_{+}$are $H K$-integrable, the primitives of $g_{-}, g_{+}$are continuous and so are uniformly continuous on $\Delta$.

Moreover, the fact that $f \in D_{\mathrm{HK}}$ also implies that the primitive of $f$ is uniformly continuous on $\Delta$.

From (12), we get

$$
\left|F u\left(x_{2}, y_{2}\right)-F u\left(x_{1}, y_{1}\right)\right| \leq c_{1} \omega(u, \varepsilon)+c_{2} \omega(u, \varepsilon), \quad \text { as } \varepsilon \rightarrow 0 .
$$

Since $u$ is an arbitrary element of $V$ in (13), we obtain

$$
\omega(F V, \varepsilon) \leq c_{1} \omega(u, \varepsilon)+c_{2} \omega(u, \varepsilon) .
$$

Hence,

$$
\lim _{\varepsilon \rightarrow 0} \sup \omega(F V, \varepsilon) \leq\left(c_{1}+c_{2}\right) \lim _{\varepsilon \rightarrow 0} \sup \omega(u, \varepsilon)
$$

It follows from (14) and Lemma 2.9 that

$$
\mu(F V)) \leq\left(c_{1}+c_{2}\right) \mu(V) .
$$

According to Lemma 2.10, $F$ has at least one fixed point in the space $B_{r}$. The proof is therefore complete.

According to Theorem 3.2 and (10) the definition of the operator $F$, we have: 
Theorem 3.3 Under the assumptions $\left(D_{1}\right)-\left(D_{5}\right), E q$. (2) has at least one solution in the space $C(\Delta)$.

\section{Application}

Example 4.1 Consider the following Darboux problem:

$$
\left\{\begin{array}{l}
\frac{\partial^{2} u}{\partial x \partial y}(x, y)=x^{2} e^{-y} \sin \frac{u(x, y)}{2}+y R^{\prime}(x)+x R^{\prime}(y), \quad(x, y) \in[0,1] \times[0,1], \\
u(x, 0)-\left(\frac{x}{4\left(1+x^{2}\right)}+\frac{u(x, y)}{4+x^{2}}\right)=\frac{1}{4+y^{2}}, \\
u(0, y)-\frac{1}{2} \arctan \left[\frac{u(x, y)}{8+\sqrt{y}}+\frac{2 y}{1+y^{2}}\right]=\frac{1}{2} \arctan x+\frac{1}{4},
\end{array}\right.
$$

where $R^{\prime}(x)$ is the distributional derivative of the Riemann function $R(x)=\sum_{n=1}^{\infty} \frac{\sin n^{2} \pi x}{n^{2}}$. With the following choices, it is evident that Eq. (16) is a special case of Eq. (2) with

$$
\begin{aligned}
& g(x, y, u(x, y))=x^{2} e^{-y} \sin \frac{u(x, y)}{2}, \\
& f(x, y)=y R^{\prime}(x)+x R^{\prime}(y), \\
& \psi_{1}(x, u)=\frac{x}{4\left(1+x^{2}\right)}+\frac{u(x, y)}{4+x^{2}}, \\
& \psi_{2}(y, u)=\frac{1}{2} \arctan \left[\frac{u(x, y)}{8+\sqrt{y}}+\frac{2 y}{1+y^{2}}\right], \\
& h_{1}(y)=\frac{1}{4+y^{2}}, \\
& h_{2}(x)=\frac{1}{2} \arctan x+\frac{1}{4}, \\
& \Delta=[0,1] \times[0,1] .
\end{aligned}
$$

Now we show that all the conditions of Theorem 3.2 are satisfied for Eq. (16).

(i) Obviously, $\psi_{i}(i=1,2)$ are continuous, and suppose that $(x, y) \in[0,1] \times[0,1]$ and $u, v \in C(\Delta)$. Then we can get the following estimate:

$$
\begin{aligned}
& \left|\psi_{1}(x, u)-\psi_{1}(x, v)\right| \leq \frac{1}{4+x^{2}}|u-v| \leq \frac{1}{4}|u-v|, \quad \text { so } c_{1}=\frac{1}{4}, \\
& \left|\psi_{2}(y, u)-\psi_{2}(y, v)\right| \leq \frac{1}{2} \cdot \frac{1}{8+\sqrt{y}}|u-v| \leq \frac{1}{16}|u-v|, \quad \text { so } c_{2}=\frac{1}{16} \\
& M_{1}=\sup \left\{\left|\psi_{1}(x, 0)\right|: x \in[0,1]\right\}=\frac{1}{8}, \\
& M_{2}=\sup \left\{\left|\psi_{2}(y, 0)\right|: y \in[0,1]\right\}=\frac{\pi}{8}
\end{aligned}
$$

(ii) Clearly, the functions $h_{i}$ are continuous, $h_{1}(0)=h_{2}(0)=\frac{1}{4}$, and

$$
M_{3}=\sup \left\{\left|h_{1}(y)\right|: y \in[0,1]\right\}=\frac{1}{4}, \quad M_{4}=\sup \left\{\left|h_{2}(x)\right|: x \in[0,1]\right\}=\frac{\pi}{8}+\frac{1}{4} .
$$

(iii) The function $f$ is $D_{\mathrm{HK}}$-integrable on $\Delta$, and

$$
M_{5}=\sup _{(x, y) \in \Delta}\left|\int_{0}^{x} \int_{0}^{y} f(s, t) d t d s\right|<2 .
$$


(iv) The function $g$ is continuous, and

$$
-x^{2} e^{-y} \preceq g(x, y, u(x, y)) \preceq x^{2} e^{-y},
$$

if we put $g_{-}(x, y)=-x^{2} e^{-y}, g_{+}(x, y)=x^{2} e^{-y}$, then we have

$$
M_{6}=\sup _{(x, y) \in \Delta}\left\{\left|\int_{0}^{x} \int_{0}^{y} g_{-}(s, t) d t d s\right|+\left|\int_{0}^{x} \int_{0}^{y} g_{+}(s, t) d t d s\right|\right\}=\frac{2}{3}-\frac{2}{3 e} .
$$

(v) It is easy to check that for each number $r \geq 5$, we have the following inequality:

$$
M_{1}+M_{2}+2 M_{3}+M_{4}+M_{5}+M_{6}+\left(c_{1}+c_{2}\right) r<r
$$

Consequently, all the conditions of Theorem 3.2 are satisfied and Eq. (16) has at least one solution in the space $C(\Delta)$.

Remark 4.2 It is well known that the function $R(x)$ given by Riemann is continuous but pointwise differentiable nowhere on $[0,1]$ (see, e.g.,[14]), then the distributional derivative $R^{\prime}(x)$ in Eq. (16) is neither $H K$ nor Lebesgue integrable. Hence, this example is not covered by any result using $H K$ or Lebesgue integral. Thus, Theorem 3.3 is more extensive.

\section{Conclusions}

In this research, by using the method associated with the technique of measure of noncompactness and the Darbo fixed points theorem, we studied the existence of solutions for the Darboux problem involving the distributional Henstock-Kurzweil integral, and we obtained the existence of at least one solution for the Darboux problem we considered.

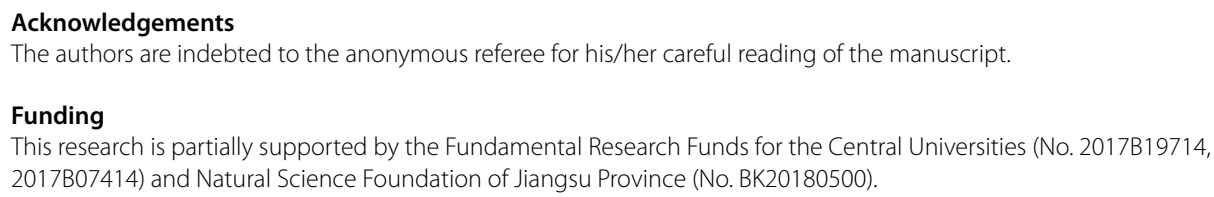

Availability of data and materials

Not applicable.

Competing interests

The authors declare that there is no conflict of interests regarding the publication of this paper.

Authors' contributions

The authors read and approved the final manuscript. All authors contributed equally to the writing of this paper.

Author details

${ }^{1}$ College of Science, Hohai University, Nanjing, China. ${ }^{2}$ Nanjing Hexi Foreign Language School, Nanjing, China.

\section{Publisher's Note}

Springer Nature remains neutral with regard to jurisdictional claims in published maps and institutional affiliations.

Received: 12 September 2018 Accepted: 15 November 2018 Published online: 22 November 2018

\section{References}

1. Bugajewski, D., Szufla, S.: On the Aronszajn property for differential equations and the Denjoy integral. Comment. Math. Prace Mat. 35, 61-69 (1995)

2. Cichoń, M., Kubiaczyk, I.: Kneser-type theorem for the Darboux problem in Banach spaces. Comment. Math. Univ. Carol. 42(2), 267-279 (2001) 
3. Majcher, P., Sharma, S.: Application of measures of weak noncompactness to a nonlocal Darboux problem. Demonstr. Math. 42(3), 521-531 (2009)

4. Lee, P.Y.: Lanzhou Lectures on Henstock Integration. World Scientific, Singapore (1989)

5. Chew, T.S., Flordeliza, F.: On $x^{\prime}=f(t, x)$ and Henstock-Kurzweil integrals. Differ. Integral Equ. 4(4), 861-868 (1991)

6. Liu, W., Ye, G., Wang, Y., Zhou, X.: On periodic solutions for first-order differential equations involving the distributional Henstock-Kurzweil integral. Bull. Aust. Math. Soc. 86(2), 327-338 (2012)

7. Schwabik, Š., Ye, G.: Topics in Banach Space Integration. World Scientific, Singapore (2005)

8. Talvila, E.: The distributional Denjoy integral. Real Anal. Exch. 33(1), 51-82 (2008)

9. Ye, G., Liu, W.: The distributional Henstock-Kurzweil integral and applications. Monatshefte Math. 181(4), 975-989 (2016)

10. Banaś, J., Goebel, K.: Measures of Noncompactness in Banach Spaces. Marcel Dekker, New York (1980)

11. Ang, D.D., Schmitt, K., Vy, L.K.: A multidimensional analogue of the Denjoy-Perron-Henstock-Kurzweil integral. Bull. Belg. Math. Soc. Simon Stevin 4, 335-371 (1997)

12. Mursaleen, M.: Differential equations in classical sequence spaces. Rev. R. Acad. Cienc. Exactas Fís. Nat., Ser. A Mat. $111(2), 587-612(2017)$

13. Kazemi, M., Ezzati, R.: Existence of solution for some nonlinear two-dimensional Volterra integral equations via measures of noncompactness. Appl. Math. Comput. 275, 165-171 (2016)

14. Hardy, G.H.: Weierstrass's non-differentiable function. Trans. Am. Math. Soc. 17(3), 301-325 (1916)

\section{Submit your manuscript to a SpringerOpen ${ }^{\circ}$ journal and benefit from:}

- Convenient online submission

- Rigorous peer review

- Open access: articles freely available online

- High visibility within the field

- Retaining the copyright to your article

Submit your next manuscript at $\gg$ springeropen.com 